\title{
EL BONO DE DESARROLLO HUMANO
}

\section{AMUEL MAGOONAL}

YTUMIUURARIIUA

A partir de septiembre de 1998, el Estado Ecuatoriano en el Gobierno de Jamil Mahuad implementó una ayuda monetaria conocido entonces, con el nombre de Bono Solidario, siendo las principales beneficiarias las madres de familia de bajos recursos y las personas de edad avanzada y con discapacidad, quienes recibían $\$ 15,10$ y $\$ 7,60$ respectivamente.

En el Gobierno de Lucio Gutiérrez recibe el nombre de Bono de Desarrollo Humano, implementando entonces una beca escolar cuya finalidad fue insertar y mantener en el sistema educativo a los hijos de las familias pobres. Para entonces las madres de familias pobres recibían $\$ 15,00$ y los ancianos y discapacitados $\$ 11,50$.

En el 2007 en el Gobierno de Rafael Correa, el Bono de Desarrollo Humano incrementa su valor a \$30,00 Para Agosto del 2009 aumenta a $\$ 35$ y en enero del 2013 a $\$ 50$ mensuales hasta la actualidad. Estos valores son entregados a madres de familia que se encuentran bajo la línea de pobreza; adultos mayores con 65 años de edad o más, que vivan en condiciones de vulnerabilidad y que no se encuentren afiliados al IESS; y, personas con discapacidad que de igual manera se encuentren en condiciones de vulnerabilidad y que no estén afiliadas al IESS.

\section{Entre los objetivos de BDH están:}

- Garantizar a las familias un nivel mínimo de consumo.

- Contribuir con la disminución de desnutrición crónica y enfermedades inmuno-reversibles en niñas y niños de hasta 5 años.

- Promover la matriculación escolar y su permanencia a los niños y niñas de 6 a 16 años

- Reducir el empleo infantil

- Cuidar y proteger al adulto mayor y personas con limitaciones físicas y mentales.

Existen diferentes causas por las que los beneficiarios de BDH pueden dejar de percibir este beneficio que otorga el gobierno, en virtud que las bases de datos son depuradas periódicamente. Las causas por las que una persona puede dejar de cobrar son varias:
- La mayoría de beneficiarios sale porque ha mejorado su índice de bienestar familiar: mayor acceso a servicios básicos, vivienda, trabajo, educación, salud, bienes, seguro social, etc.

- El Bono de Desarrollo Humano es para jefas de hogar con hijos de hasta 18 años. Si sus hijos han superado esta edad es posible que sea eliminada de la base de datos del Registro Social.

- Personas que ahora han pasado a recibir el Bono Joaquín Gallegos Lara.

- Quienes no cumplan con el compromiso de corresponsabilidad (llevar a los niños a la escuela y al Centro de Salud).

- El fallecimiento es otra causa de exclusión.

En la siguiente tabla se puede observar el número de personas que se encuentran habilitadas para recibir el $\mathrm{BDH}$, por provincia y tipo de beneficiario durante el primer semestre de este año.

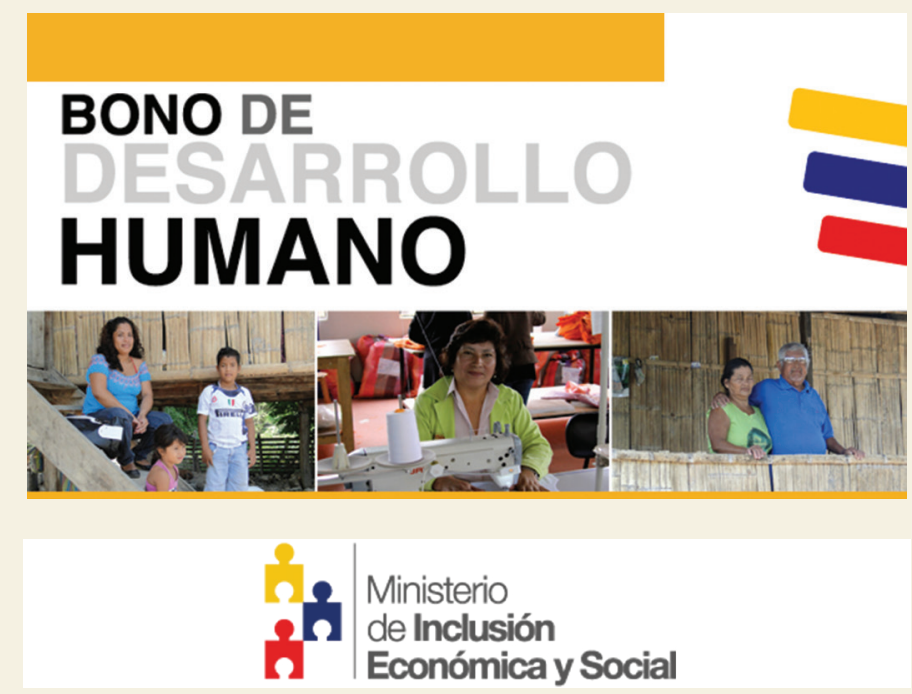


Tabla 1. PERSONAS HABILITADAS AL PAGO DEL BONO DE DESARROLLO A NIVEL NACIONAL PRIMER SEMESTRE 2014

\begin{tabular}{|c|c|c|c|c|}
\hline PROUNMGAS & Madines & $\begin{array}{l}\text { Adulitos } \\
\text { Mayones }\end{array}$ & $\begin{array}{l}\text { Persones con } \\
\text { Diseapribiliand }\end{array}$ & $\begin{array}{l}\text { Menores gon } \\
\text { Diseraprabidad }\end{array}$ \\
\hline AZUAY & 41051 & 33090 & 4822 & 4822 \\
\hline BOLIVAR & 22968 & 14634 & 1657 & 1657 \\
\hline CAÑAR & 17637 & 13149 & 1501 & 1501 \\
\hline $\mathrm{CARCHI}$ & 11682 & 9646 & 1271 & 1271 \\
\hline CHIMBORAZO & 41934 & 29061 & 3384 & 3384 \\
\hline COTOPAXI & 41123 & 24661 & 2399 & 2399 \\
\hline EL ORO & 33390 & 25545 & 4854 & 4854 \\
\hline ESMERALDAS & 53791 & 18964 & 3799 & 3799 \\
\hline FCO DE ORELLANA & 13905 & 3561 & 908 & 908 \\
\hline GALAPAGOS & 223 & 316 & 45 & 45 \\
\hline GUAYAS & 240409 & 117056 & 21376 & 21376 \\
\hline IMBABURA & 28911 & 20272 & 2510 & 2510 \\
\hline LOJA & 37132 & 30334 & 3961 & 3961 \\
\hline LOS RIOS & 86380 & 38019 & 4915 & 4915 \\
\hline MANABI & 150095 & 70633 & 12867 & 12867 \\
\hline MORONA SANTIAGO & 14649 & 4832 & 901 & 901 \\
\hline NAPO & 10565 & 3152 & 818 & 818 \\
\hline PASTAZA & 5922 & 2423 & 466 & 466 \\
\hline $\mathrm{PICHINCHA}$ & 52553 & 46395 & 7759 & 7759 \\
\hline SANTA ELENA & 29857 & 12454 & 2229 & 2229 \\
\hline $\begin{array}{l}\text { SANTO DOMINGO DE } \\
\text { LOS TSACHILAS }\end{array}$ & 28380 & 13660 & 2569 & 2569 \\
\hline SUCUMBIOS & 17322 & 5210 & 1304 & 1304 \\
\hline TUNGURAHUA & 32947 & 26748 & 2708 & 2708 \\
\hline ZAMORA CHINCHIPE & 8981 & 3875 & 887 & 887 \\
\hline ZONA NO DELIMITADA & 4307 & 1454 & 215 & 215 \\
\hline TOTALES & 1026114 & 569144 & 90125 & 90125 \\
\hline
\end{tabular}

Fuente: Ministerio de Inclusión Económica y Social, Distrito MIES - AMBATO Elaboración: OBEST.

En la figura 1 se observa las 11 provincias que tienen mayor número de personas habilitadas para el cobro del $\mathrm{BDH}$.

Guayas es la provincia con mayor número de habilitados con $22.5 \%$ respecto al total nacional, esto significa un gasto de 19.340.550 USD al gobierno por el BDH, seguida de Manabí $13.8 \%$ de habilitados, Los Ríos 7.6\%, Pichincha 6.4\%, Esmeraldas $4.6 \%$, Chimborazo 4.4\%, Cotopaxi 4\%, El Oro 3.8\%, en onceavo lugar aparece Tungurahua con $3.7 \%$, que representa un beneficio de 3.146.200 USD para las personas habilitados en nuestra provincia, finalmente el $20.3 \%$ se dividen el resto de las provincias del País, la entrega del BDH tiene relación directamente proporcional al nivel de pobreza de la Provincia.
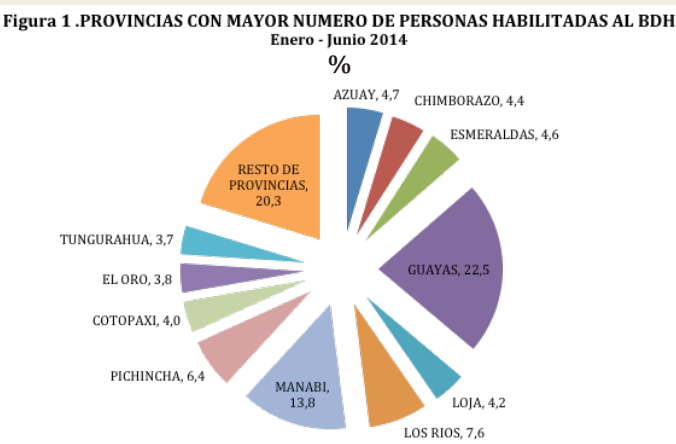

Fuente: Ministerio de Inclusión Económica y Social, Distrito MIES - AMBATO Elaboración: OBEST.

En la figura 2 se observa que las madres de familia de bajos recursos son las mayores habilitadas del BDH con $60 \%$, seguido de los adultos mayores 33\%, personas con discapacidad 5\% y finalmente menores de edad con discapacidad el $2 \%$.

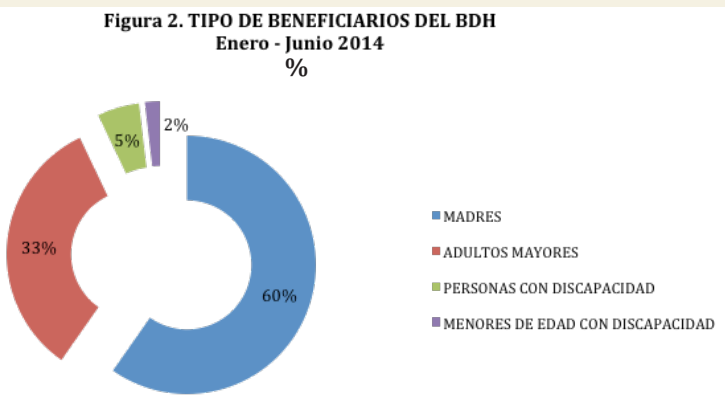

Fuente: Ministerio de Inclusión Económica y Social, Distrito MIES - AMBATO Elaboración: OBEST.

Según datos del INEC, la proyección de la población en este año es de 16'027.466 habitantes y con los datos proporcionados por el MIES se puede determinar que el $10,7 \%$ de la población total se encuentra habilitada para la obtención del BDH en lo que va del año y de igual manera sucede en Tungurahua en donde el 11,4\% se encuentra habilitada con respecto a la población total de la provincia.
Figura 3. POBLACIÓN TOTAL Y BENEFICIARIOS BDH NACIONAL Y TUNGURAHUA 2014 (miles de personas)

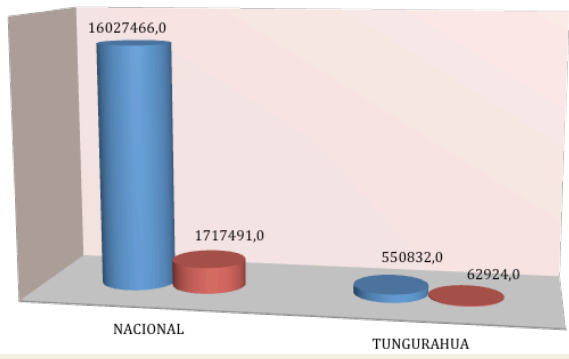

- POBLACION TOTAL

- POBLACIÓN HABILITADA

Fuente: Ministerio de Inclusión Económica y Social, Distrito MIES - AMBATO Elaboración: OBEST. 
En comparación al año anterior se puede determinar que el porcentaje de habilitados al BDH ha disminuido el 18\% en madres de bajos recursos, en adultos mayores disminuyo en $3.5 \%$, en personas con discapacidad se incrementó en $2.5 \%$ y en menores de edad con discapacidad su incremento fue del $15.8 \%$.

\section{Figura 4. COMPARACIÓN BENEFICIARIOS DEL BDH EN TUNGURAHUA \\ $2013-2014$ \\ (miles de personas)}

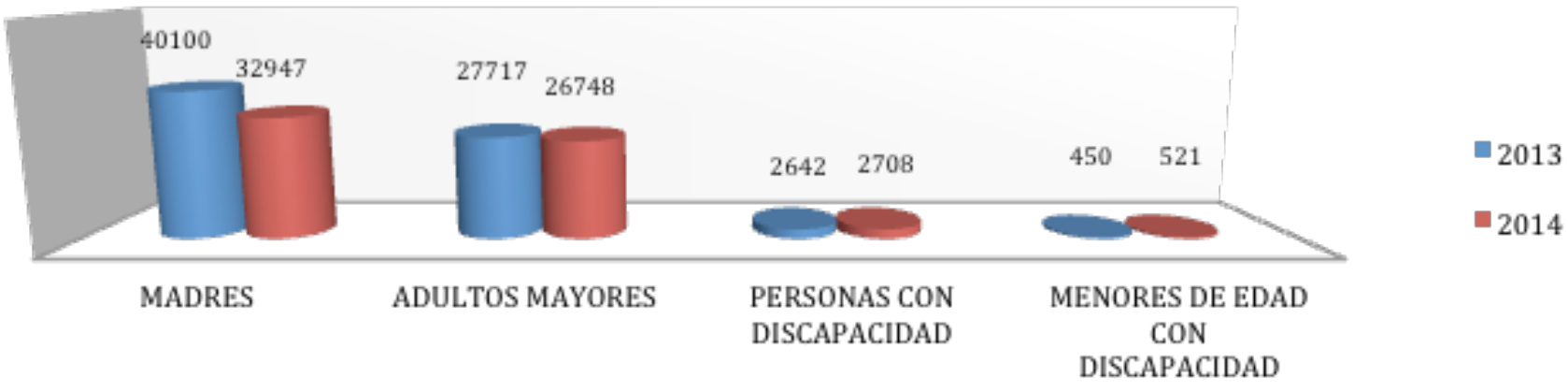

Fuente: Ministerio de Inclusión Económica y Social, Distrito MIES - AMBATO

Elaboración: OBEST.

En Tungurahua 32947 madres se benefician con el BDH es decir $52 \%$ de la totalidad de habilitados de la provincia, seguido de 26748 adultos mayores 43\%, 2708 personas con discapacidad $4 \%$ y finalmente 521 menores de edad con discapacidad tan solo $1 \%$.

Figura 5. BENEFICIARIOS BDH EN TUNGURAHUA ENERO - JUNIO 2014

$\%$

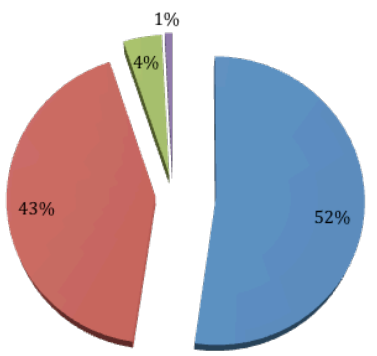

MADRES

- ADULTOS MAYORES

PERSONAS CON DISCAPACIDAD

MENORES DE EDAD CON DISCAPACIDAD

Fuente: Ministerio de Inclusión Económica y Social, Distrito MIES - AMBATO

Elaboración: OBEST.
El BDH es uno de los pilares del sistema de protección social de nuestro país, los beneficiarios en cada una de las provincias logran tener un mejor acceso a la alimentación, salud o educación, priorizando las necesidades de cada familia, además que con esta ayuda se busca reducir el trabajo infantil y mejorar la calidad de vida de la tercera edad y personas con limitaciones físicas o mentales, este tipo de programas son experiencias extendidas en América Latina pues los organismos internacionales se han encargado de sostenerlos y aplaudirlos desde sus estudios de impacto.

\section{Referencias}

Ministerio de Inclusión Económica y Social, Distrito MIES - AMBATO

Observatorio Económico y Social de Tungurahua (OBEST) - Universidad Técnica de Ambato.

Econ. Tatiana Vayas

Lic. Sandra Garcés 